\title{
Can Radiation Enhance Response to Checkpoint Inhibition in Advanced-Stage NSCLC? A Case Series and Review of the Literature
}

\author{
Shraddha M Dalwadi ${ }^{1}$, Hector A Preti ${ }^{2}$, Stephen B Chiang ${ }^{2}$, E Brian Butler ${ }^{2}$, Bin S The ${ }^{2}$ and Andrew M \\ Farach $^{2 *}$
}

${ }^{1}$ Baylor College of Medicine, Houston, TX, USA

${ }^{2}$ Houston Methodist Hospital, Houston, TX, USA

*Corresponding author: Andrew M Farach, Department of Radiation Oncology, Houston Methodist Hospital, 6565 Fannin St. Houston, TX, USA, Tel: 713-441-4812; Fax: 713-441-4493; E-mail: amfarach@ @oustonmethodist.org

\begin{abstract}
Introduction: The abscopal effect is an elusive phenomenon first described by Mole in 1953 as tumor regression observed at sites distant to an irradiated area. Anti-PDL1 monoclonal antibodies (such as nivolumab) were recently approved as first and second-line treatment for advanced-stage NSCLC, but overall response rates remain low for these patients. Herein, we report two cases of stage IV lung adenocarcinoma that achieved complete response after the addition of radiation therapy to an existing anti-PD-1 regimen.

Methods: Retrospective chart review of two patients was conducted, followed by systematic literature review using PubMed for abscopal effect observed in lung adenocarcinoma.

Results: Two patients with widely metastastic lung adenocarcinoma and progression on multimodal therapy experienced complete resolution of radiographic disease after palliative radiotherapy administered in combination with maintenance anti-PD-1 therapy.

Conclusion: Few studies describe the abscopal phenomenon in lung cancer. Adjunct radiotherapy may boost response rates in advanced-stage NSCLC managed with checkpoint inhibition via the abscopal effect. Investigative efforts are necessary to examine combined immunoradiotherapy in metastatic NSCLC.
\end{abstract}

Keywords: Lung neoplasms; Radiotherapy; Immunotherapy

Received Date: March 04, 2018; Accepted Date: March 18, 2018; Published Date: March 25, 2018

\section{Introduction}

The abscopal (ab-scopus, or "away from the target") effect is an elusive phenomenon first described by Mole in 1953 as tumor regression observed at sites distant to an irradiated area [1]. Most theories discussing underlying mechanisms of this phenomenon focus on host immune activation, including the production of anti-tumor $\mathrm{T}$ lymphocytes, alteration of tumor microenvironment, and generation of a pro-inflammatory state [2]. While radiotherapy may be efficient at releasing tumor antigens; cancer-induced immunosuppression and immunoevasion prevent the development of an adequate systemic antitumor immune response [3,4].

Citation: Farach AM. Can Radiation Enhance Response to Nivolumab in Advanced-Stage NSCLC? A Case Series and Review of the Literature. J Clin Cases Rep 2018: 1(1) 23-29. DOI: https://doi.org/10.46619/joccr.2018.1-1006 
www.tridhascholars.org | April-2018

Immune checkpoint inhibitors act to suppress immune-inhibitory signals that cancer cells utilize for immune escape and have shown considerable therapeutic promise [5]. Nivolumab and pembrolizumab are novel monoclonal antibodies that act by suppressing PD-1 receptors and boosting immune response [5]. PD-1 (Programmed Cell Death 1) is a surface protein found on T-lymphocytes that binds to PD-L1 (Programmed Cell Death Ligand 1) causing inactivation. Normally, this tempers the host immune system to prevent overreaction and autoimmunity. Many cancer cells however produce PDL-1 as a form of immunoevasion, especially melanomas, placental tissues, and respiratory epithelial tumors [6]. PD-1 inhibitors diminish immunoregulatory signals by preventing PD-1 from binding to tumor-cell generated PD-L1, thus allowing T-lymphocytes to function normally.

Nivolumab is approved as a second-line treatment in non-small cell lung carcinoma (NSCLC) and pembrolizumab is approved as a first-line treatment in metastatic NSCLC with $>50 \%$ PD-L1 expression or in patients with progression after platinum-containing chemotherapy and at least $1 \%$ of cells expressing PD-L1 [7]. While evidence demonstrates the utility of checkpoint inhibitors in advanced-stage NSCLC unresponsive to standard of care chemotherapy, overall response rates remain low for these patients at around twenty percent [8,9]. Following the radiation therapy, reports of dramatic responses in patients previously unresponsive to immune checkpoint monotherapy, indicating a potential synergistic effect of combined immunoradiotherapy [10]. In fact, a recent report demonstrated dramatically increased progression free survival and overall survival in patients treated with a PD-1 inhibitor who had previously received extra thoracic radiation therapy [11]. Although a combined approach may enhance abscopal effects, the therapeutic use of checkpoint inhibitors in conjunction with radiotherapy is limited outside of investigational protocols. Herein, we report the first two cases of serendipitous abscopal effect observed in metastatic lung adenocarcinoma after treatment with a PD-1 inhibitor and palliative radiation.

\section{Case Series}

\section{Patient A}

In June 2014, a 75-year-old woman was diagnosed with cT4N3M0 stage IIIB lung adenocarcinoma with bilateral mediastinal and supraclavicular lymphadenopathy. She initially received two cycles of induction carboplatin and placitaxel with partial response followed by 66 Gy concurrent chemoradiation. She achieved remission until October 2015, at which time PET/CT demonstrated recurrence in an AP window and left hilar lymph node outside of the previous radiation field, peri-portal adenopathy, a left adrenal metastasis, a lingular nodule, and a pericardial nodule. Nivolumab therapy was initiated biweekly with 35 Gy radiation in 14 fractions to the symptomatic mediastinal recurrence. Repeat PET/CT in January 2016 demonstrated a dramatic complete resolution of all disease except an adrenal metastasis, indicative of an abscopal response. The single adrenal metastasis was treated to resolution with stereotactic body radiation therapy, 30 Gy in 5 fractions. A subsequent PET/CT demonstrated a complete metabolic response. The patient continued to do well with no evidence of disease on maintenance nivolumab monotherapy until symptomatic progression in April 2016. She ultimately deceased due to cancer-related complications in May 2016. A detailed clinical timeline and relevant imaging is supplied in Figure 1A and $1 \mathrm{~B}$ respectively. 


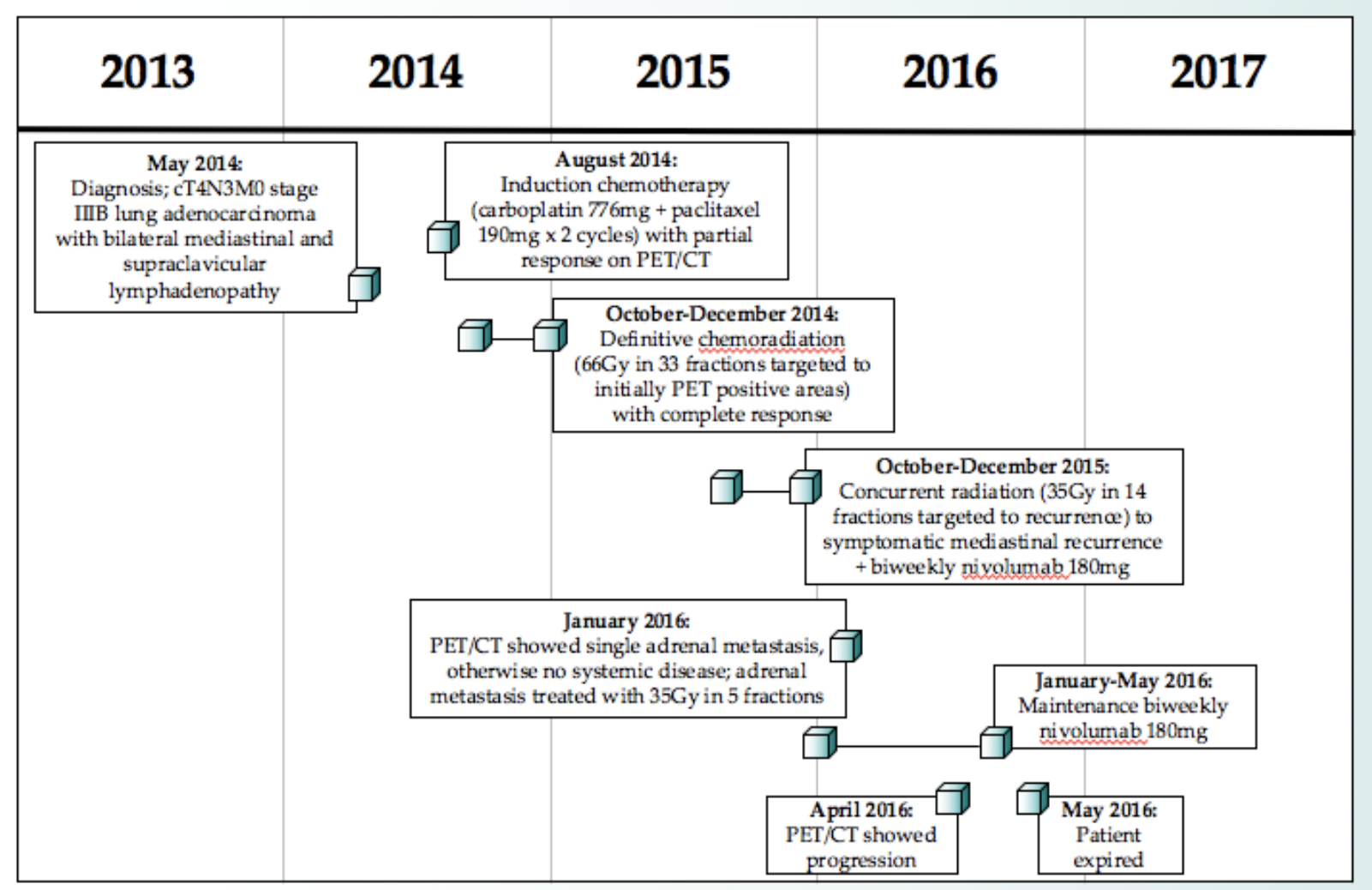

Figure 1A: Patient A clinical timeline.
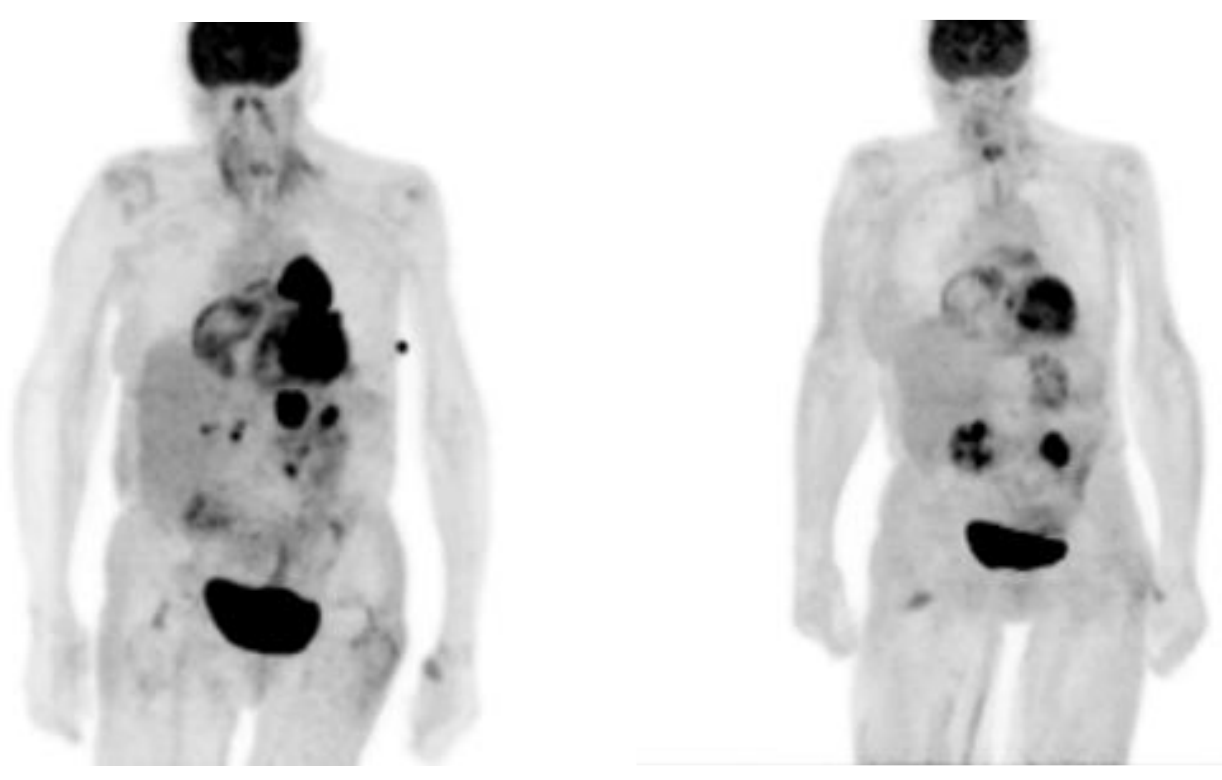

Figure 1B: Patient A Pre- and Post-XRT PET/CT.

\section{Patient B}

In July 2012, a 32-year-old woman presented with cT2N3M1 stage IV adenocarcinoma of the lung with osseous and brain metastases. Initially, she achieved 2 years' remission after concurrent carboplatin, placitaxel, and bevacizumab chemotherapy with 30 Gy of palliative radiation to 3 brain nodules. Her maintenance chemotherapy during that time was erlotinib given her positive EGFR receptor status. However, PET/CT in July 2014 demonstrated choroidal, chest, liver, adrenal, brain, and 
www.tridhascholars.org | April-2018

osseous uptake. Over the following 1.5 years, her metastatic burden persisted despite aggressive systemic therapy. She was initially placed on carboplatin and paclitaxel chemotherapy. She also required palliative radiation to multiple targets throughout disease progression (41.4 Gy to the bilateral choroid, 35 Gy to the sacrum, 30 Gy to the whole brain, 30 Gy to the thoracic spine, $20 \mathrm{~Gy}$ to the lumbar spine, $30 \mathrm{~Gy}$ to the sternum, 30 Gy to the shoulder, 20 Gy to the bilateral knees, and 20 Gy to the femur). Due to persistent disease, she was considered a good candidate for ipilimumab and nivolumab immunotherapy.

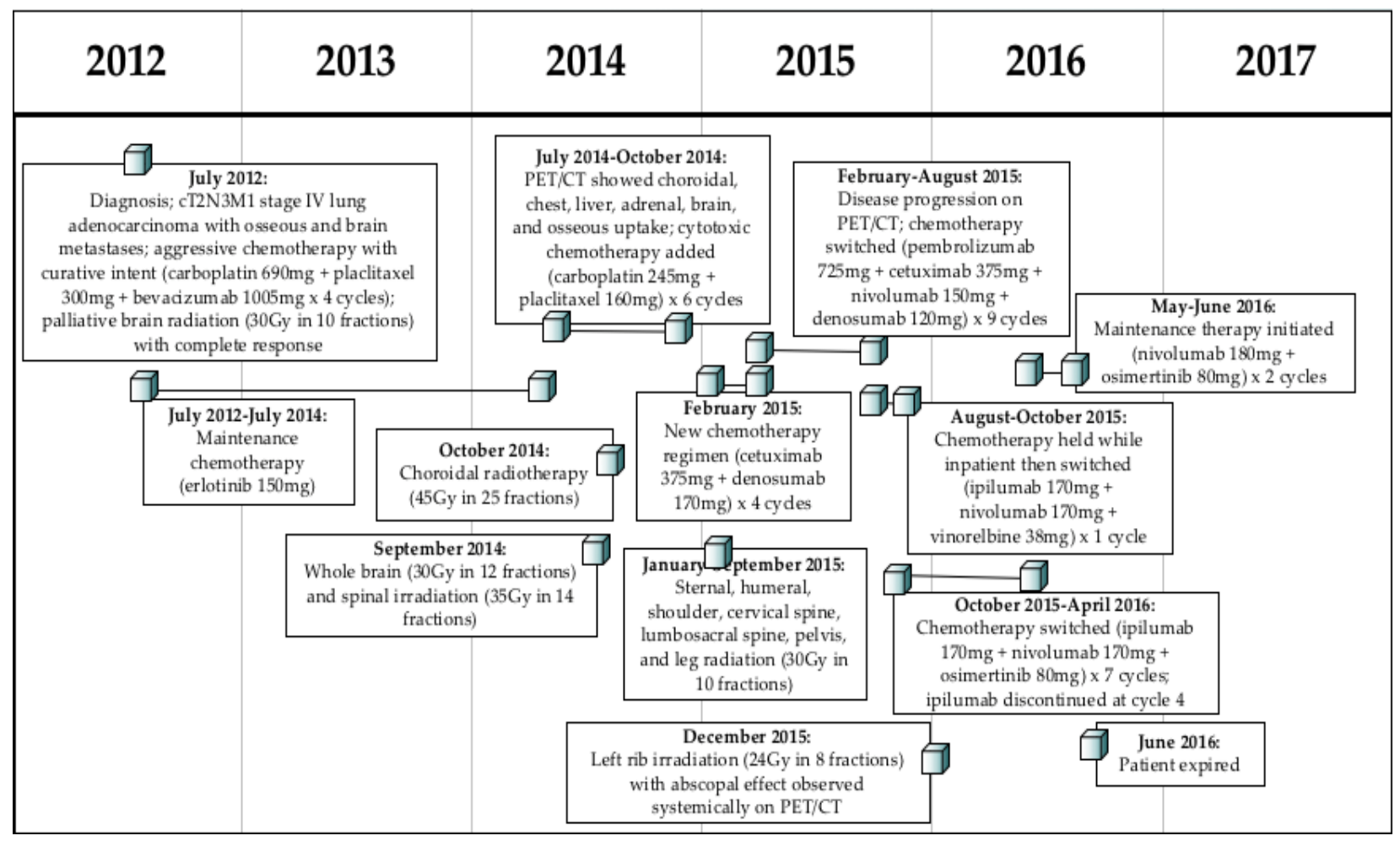

Figure 2A: Patient B clinical timeline.
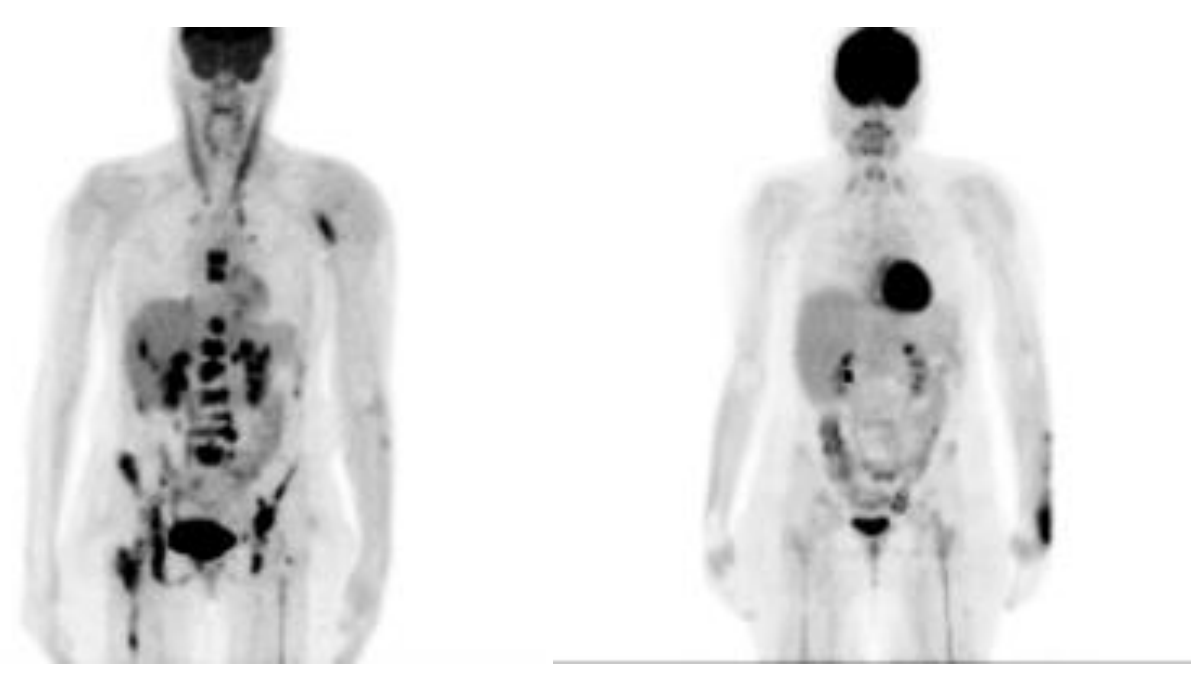

Figure 2B: Patient B Pre- and Post-XRT PET/CT. 
www.tridhascholars.org | April-2018

Interval imaging revealed poor response to therapeutic intervention until a painful left rib metastasis was radiated with 24 Gy over 8 fractions. PET/CT done in March 2016 after rib radiation showed complete metabolic response throughout the body almost 2 years after initial recurrence and 4 years after presentation, evidencing the synergistic effect of tumor cell radiolysis on immunomodulation even in a previous non-responder. Unfortunately, the patient developed resolute metastases in the chest, liver, pancreas, and bone and succumbed to cancer-related complications in June 2016. Relevant clinical timeline and imaging are shown in Figures $2 \mathrm{~A}$ and $2 \mathrm{~B}$ respectively.

\section{Discussion}

Our experience with two lung adenocarcinoma patients treated with combined immunoradiotherapy shows that systemic resolution of high metastatic burden is possible, even in previously unresponsive patients. The literature supports the notion that NSCLC could be more susceptible to the synergistic effects of radiation and immune therapy than previously thought. One study showed that low mediastinal recurrence rates in NSCLC patients treated with stereotactic body radiotherapy (SBRT) could not be accounted for by incidental mediastinal dose alone, implying that immunologic tumor cell death occurs distinct from ablative irradiation even in patients not receiving immunomodulation [12]. A variety of reports evidence NSCLC to exhibit an abscopal response at distant sites as well (Table 1), further suggesting that radiation augments host immune response to cancer and could be susceptible to enhancement by immunomodulatory therapy.

Still, many uncertainties surround this approach. Although we achieved success with non-ablative radiation, the dose necessary to achieve the optimal immune response is debated [13]. In vitro studies show radiotherapy to have chemotherapeutic synergy in achieving immunogenic cell death in a dose-dependent manner, proposing ablative doses to be more effective [14]. Furthermore, one prospective randomized trial showed that ipilimumab with non-ablative bone-directed radiotherapy was not significantly different from a placebo [15]. Yet another study found that hypo fractionated, but not single-dose, radiotherapy induces response when combined checkpoint inhibitor therapy [16]. While our experience is that non-ablative doses are sufficient, the increased use of SBRT in oligometastatic disease warrants consideration of whether or not high-dose fractionation is more likely to achieve adequate tumor cell radiolysis and antigen release.

\begin{tabular}{|c|c|c|c|c|c|c|c|}
\hline Investigator & Age & Sex & Histology & Stage & XRT Location & $\begin{array}{c}\text { Other } \\
\text { Concurrent } \\
\text { Therapies }\end{array}$ & Comments \\
\hline Golden [17] & 64 & $\mathrm{M}$ & Adenocarcinoma & IV & Spine & $\begin{array}{c}\text { Ipilimumab } \\
\text { Explored the efficacy of phased } \\
\text { vs. concurrent regimen }\end{array}$ \\
\hline Yarchoan [18] & 60 & $\mathrm{M}$ & Adenocarcinoma & IV & Brain & $\begin{array}{c}\text { Cisplatin+ } \\
\text { Pemetrexed }\end{array}$ & $\begin{array}{c}\text { Proposes oligometastatic state } \\
\text { as optimal time to treat }\end{array}$ \\
\hline Siva [19] & 78 & $\mathrm{~F}$ & Adenocarcinoma & IV & $\begin{array}{c}\text { Adrenal Gland, } \\
\text { Humerus }\end{array}$ & None & No concurrent immunotherapy \\
\hline Yoon [20] & 72 & $\mathrm{M}$ & Unclassifiable & III & Lung & $\begin{array}{c}\text { Oncothermia, } \\
\text { GM-CSF }\end{array}$ & XRT directed a primary site \\
\hline Rees [21] & 56 & $\mathrm{M}$ & Adenocarcinoma & IV & Lung & Unknown & XRT directed a primary site \\
\hline Kodama [22] & 74 & $\mathrm{M}$ & Adenocarcinoma & II & Regional LN & BCG-CWS & $\begin{array}{c}\text { Considers applicability to } \\
\text { locally advanced disease }\end{array}$ \\
\hline
\end{tabular}

Table 1: Case reports describing the abscopal effect in lung cancer.

Recurrent metastatic NSCLC carries an abysmal prognosis with historical median survival of 4 months with third-line treatment [17]. A complete response to third-line chemotherapy is exceedingly rare. While it is difficult to state that 
www.tridhascholars.org | April-2018

prognosis was improved for the presented patients, the drastic improvement in tumor burden demonstrates a potential for prolonged remission if a sustained response can be obtained. The short-lived remission in the presented cases demonstrates the need for investigations dedicated to understanding the abscopal effect in practice and enhancing the durability of treatment response. Little is known about appropriate treatment sequencing, dosing schedules, patient selection, and maintenance options in combination immunoradiotherapy. This report of dramatic clinical response hopes to reveal innovative therapeutic options for patients with treatment-resistant advanced-stage NSCLC and encourage future research on harnessing the abscopal effect clinically.

\section{Conclusion}

We report two cases of metastatic lung adenocarcinoma with complete systemic resolution after treatment with combined radiation therapy and PD-1 inhibition. While promising, the preliminary successes of combined immunoradiotherapy in advanced NSCLC warrant further investigation on patient selection, toxicity, treatment sequencing, adequate radiation dose, and maintenance therapy.

\section{References}

1. Mole RH (1953) Whole body irradiation-radiobiology or medicine? The British Journal of Radiology 26(305): 234241.

2. Chen AC, Butler EB, Lo SS, et al. (2015) Radiotherapy and the abscopal effect: insight from the past, present, and future. Journal of Radiation Oncology 4(4): 321-330.

3. Formenti SC and Demaria S (2013) Combining radiotherapy and cancer immunotherapy: a paradigm shift. Journal of the National Cancer Institute 105: 256-265.

4. Ma Y, Kepp O, Ghiringhelli F, et al. (2010) Chemotherapy and radiotherapy: cryptic anticancer vaccines. Seminars in Immunology 22(3): 113-124.

5. Pardoll DM (2012) The blockade of immune checkpoints in cancer immunotherapy. Nature Reviews Cancer 12(4): 252-264.

6. Johnson DB, Peng C, Sosman JA (2015) Nivolumab in melanoma: latest evidence and clinical potential. Therapeutic Advances in Medical Oncology. 7(2): 97-106.

7. Sundar R, Cho BC, Brahmer JR, et al. (2015) Nivolumab in NSCLC: latest evidence and clinical potential. Therapeutic Advances in Medical Oncology 7(2): 85-96.

8. Barbara M, Chu Q, Juergens R, et al. (2016) Pointed progress in second-line advanced non-small-cell lung cancer: the rapidly evolving field of checkpoint inhibition. Journal of Clinical Oncology 34(14): 1676-1688.

9. Borghaei H, Paz-Ares L, Horn L, et al. (2015) Nivolumab versus docetaxel in advanced nonsquamous non-smallcell lung cancer. New England Journal of Medicine 373(17): 1627-1639.

10. Deng L, Liang H, Burnette B, et al. (2014) Irradiation and anti-PD-L1 treatment synergistically promote antitumor immunity in mice. The Journal of Clinical Investigation 124(2): 687-695.

11. Shaverdian N, Lisberg AE, Bornazyan K, et al. (2017) Previous radiotherapy and the clinical activity and toxicity of pembrolizumab in the treatment of non-small-cell lung cancer: a secondary analysis of the KEYNOTE-001 phase 1 trial. The Lancet Oncology 18(7): 895-903. 
www.tridhascholars.org | April-2018

12. Rwigema JM, Chen AM, Wang PC, et al. (2014) Incidental mediastinal dose does not explain low mediastinal node recurrence rates in patients with early-stage NSCLC treated with stereotactic body radiotherapy. Clinical Lung Cancer 15(4): 287-293.

13. Farach A, Farach-Carson MC, Butler EB, et al. (2015) The role of combined radiation and immunotherapy in breast cancer treatment. Journal of Radiation Oncology 4(4): 347-354.

14. Golden EB, Frances D, Pellicciotta I, et al. (2014) Radiation fosters dose-dependent and chemotherapy-induced immunogenic cell death. Oncoimmunology 3(4): e28518.

15. Eugene DK, Drake CG, Scher HI, et al. (2014) Ipilimumab versus placebo after radiotherapy in patients with metastatic castrationresistant prostate cancer that had progressed after docetaxel chemotherapy (CA184-043): a multicentre, randomised, double-blind, phase 3 trial. The Lancet Oncology 15(7): 700-712.

16. Dewan MZ, Galloway AE, Kawashima N, et al. (2009) Fractionated but not single-dose radiotherapy induces an immune-mediated abscopal effect when combined with anti-CTLA-4 antibody. Clinical Cancer Research 15(17): 5379-5388.

17. Golden EB, Demaria S, Schiff PB, et al. (2013) An abscopal response to radiation and ipilimumab in a patient with metastatic non-small cell lung cancer. Cancer Immunology Research 1(6): 365-372.

18. Yarchoan M, Lim M, Brahmer JR, et al. (2015) Oligometastatic adenocarcinoma of the lung: a therapeutic opportunity for long-term survival. Cureus 7(12): e409.

19. Shankar S, Callahan J, MacManus MP, et al. (2013) Abscopal effects after conventional and stereotactic lung irradiation of non-small-cell lung cancer. Journal of Thoracic Oncology 8(8): e71-e72.

20. Yoon SM and Jung SL (2012) Case of abscopal effect with metastatic non-small-cell lung cancer. Oncothermia Journal 5: 53-57.

21. Rees GJG and Ross CMD (1983) Abscopal regression following radiotherapy for adenocarcinoma. The British Journal of Radiology 56(661): 63-66.

22. Kodama K, Higashiyama M, Okami J, et al. (2014) A possible abscopal effect of post-irradiation immunotherapy in two patients with metastatic lung tumors. International Cancer Conference Journal. 3(2): 122-127.

23. Girard N, Jacoulet P, Gainet M, et al. (2009) Third-line chemotherapy in advanced non-small cell lung cancer: identifying the candidates for routine practice. Journal of Thoracic Oncology 4(12): 1544-1549. 